\title{
Chylothorax Consecutive Heartworm Disease in Dog
}

\author{
ADRIAN STANCU ${ }^{1}$, ALINA GHISE ${ }^{2}$, MARIUS PENTEA ${ }^{1}$, DELIA MIRA BERCEANU VADUVA², DANA EMILIA VELIMIROVICI ${ }^{2}$, \\ LILIANA CARPINISAN ${ }^{1}$, ROMEO T. CRISTINA ${ }^{1}$ \\ ${ }^{1}$ University of Agricultural Sciences and Veterinary Medicine, Faculty of Veterinary Medicine Timisoara, 119 Calea Aradului, \\ 300645, Timisoara, Romania \\ ${ }^{2}$ Victor Babes University of Medicine and Pharmacy Timisoara, 2 Eftimie Murgu Sq., 300041, Timisoara, Romania
}

Chylothorax was diagnosed in a Afghan dog 3yearold, thatpresented in the necropsya accumulation of lymphatic fluid in the chest cavity. Also to examine the right heart was observed to this heartworms - Dirophylaria immitis.

Keywords: chylotorax, dog, chest cavity, Dirophylaria immitis

Chylothorax is defined as the accumulation of chyle in the pleural space, which distinguishes it from the presence of pus (pyothorax) or air (pneumothorax).

Chylothorax develops when chyle, fluid containing a high concentration of chylomicrons and lymph, effuses from the thoracic duct-cisterna chyli system into the pleural space. Chylothorax is fairly uncommon in dogs and cats, is usually secondary to other conditions or diseases, and can be difficult to treat.

Chylous effusion classically has been described as a milky-white pleural fluid that fails to become clear upon centrifugation. Pseudochylous effusion is also milky white, resembles chylous effusion grossly, and typically is associated with chronic inflammatory thoracic disease. Pseudochylous effusions do not contain chylomicra, clear upon centrifugation, and contain cholesterol and proteinlecithin compounds, giving them a higher cholesterol content than serum $[1,2,9]$.

\section{Pathophysiology}

Dietary lipids, including triglycerides, cholesterol and phospholipids, undergo four major processes of assimilation. The first stage, emulsification, breaks up the lipids into smaller droplets.Emulsification starts in the stomach and continues into the small intestine. Stomach activity breaks the lipid globules into smaller droplets which can pass easily into the duodenum. Once the lipids are in the small intestine, bile acids coat and decrease the surface tension of lipid droplets, which further reduces the size of the emulsified droplets.

The second stage, hydrolysis, occurs in the jejunum. Pancreatic enzymes such as lipase, co-lipase, cholesterol esterase and phospholipase degrade lipids in the emulsified droplets into nonesterified fatty acids, monoglycerides, cholesterol and lysophospholipids. In the third stage, micelle formation, the products of hydrolysis combine with bile acids and phospholipids to form micelles, which are water-soluble. The fourth stage is absorption, during which the micelles diffuse across the apical membranes of enterocytes in the jejunum. The bile acids do not enter the enterocyte, but are reabsorbed in the ileum. Once inside the enterocytes, the lipids are re-esterified into triglycerides and phospholipids. The re-esterified lipids then combine with cholesterol, other lipids and proteins to form chylomicrons [3, 6-8].

Chylomicrons are small, water-soluble structures with a core of hydrophobic, nonpolar lipids and an outer coat of polar lipids. The chylomicrons are then absorbed into intestinal lymphatics, which travel to the cisterna chyli. The thoracic duct carries the chylomicrons from the cisterna chyli into the venous circulation for distribution to tissues [10].

Chylous effusions may result from leakage of chyle from intact lymph vessels or thoracic duct perforation. Obstruction of lymphatic flow commonly causes lymphatic hypertension, dilation of lymphatics, and subsequent leakage of lymph. Chylothorax may be idiopathic in cats, but several causes have been implicated including hypertrophic cardiomyopathy, thoracic and mediastinal neoplasia (e.g. lymphoma), mediastinal fungal granulomas (e.g. cryptococcus), heartworm disease, congenital lymphatic defects, trauma, chronic vomiting or coughing, or rupture of lymphatic vessels, diaphragmatic hernia, lung lobe torsion, and venous thrombi. Rupture of the thoracic duct secondary to trauma was once thought to be the sole cause of chylothorax, but it has been shown that the thoracic duct remains intact in the majority of patients $[6$, $8,10]$.

The thoracic duct is a lymphatic vessel that carries chyle from the intestines and into the chest where it empties into the bloodstream. Many of the conditions that cause chylothorax do so because they increase pressure in the blood vessels into which the thoracic duct empties. This increased pressure causes the chyle to back up in the duct and many new lymphatics are formed trying to bypass this obstruction. Because these lymphatics are very thin walled, they leak when there is pressure on them. Thus, the chyle seeps from the vessels and accumulates in the chest cavity. One of the most important aspects of treating chylothorax is to determine the underlying cause so that the treatment can be tailored to the cause. Unfortunately, in manyanimals the underlying cause is not determined and the condition is termed idiopathic. Some of the causes of chylothorax that have been recognized in dogs include $[5,11,13]$ :

- Mediastinal masses (lymphosarcoma, thymoma). These masses grow in the mediastinum or space between the left and right sides of the lungs. As they grow, they may partially occlude the blood vessels that the thoracic duct empties into.

-Congenital heart disease (tetralogy of Fallot, tricuspid dysplasia). Because these diseases increase pressure in the heart and the blood vessels that the thoracic duct empties into, they also cause lymph to back-up in the thoracic duct. As with mediastinal masses, new lymphatics form and these new vessels simply leak chyle into the chest cavity.

\footnotetext{
*email:alinaghise@yahoo.com; Phone: (+40)256277095
} 
-Cardiomyopathy. This is an acquired heart disease where the heart does not contract normally. Similar to congenital heart disease, this leads to an increase in pressure in the blood vessels and subsequent leakage of chyle into the chest cavity.

-Pericardial effusion. This is an accumulation of fluid within the sac that surrounds the heart. When fluid accumulates here, it causes an increase in pressure in the blood vessels that the thoracic duct normally empties into.

- Heartworm infection. Heartworm infection may cause heart failure that increases pressures in the vessels that the thoracic duct empties into.

-Fungal granulomas. These abnormal collections of cells and fungal organisms may block the vessels that they thoracic duct empties into.

-Venous thrombi. Blood clots may block the vessels that the thoracic duct empties into. This can occur after catheters have been placed in the jugular vein. It has particularly been noted when liquid diets are placed into the vein.

-Congenital abnormalities of the thoracic duct. Abnormalities of the thoracic duct have been described in Afghan hounds and may account for their developing this disease more commonly than most other breeds.

-Trauma is an uncommon cause of chylothorax in dogs; however, ithas been recognized after automobile accidents or severe vomiting. Chylothorax associated with trauma usually has a good prognosis as the thoracic duct heals fairly quickly on its own without surgery [ $7,12,14-16]$.

\section{Experimental part}

\section{Materials and methods}

It was performed an 3 years old Afghan dog postmortem examination, following a sudden death. The autopsy was performed according to specific techniques mammals.

The corpses are placed in favorable positions for necropsy (dorsal, lateral) with their four limbs perfectly secured. For a correct skinning, one should perform a long incision, starting with the submandibular midline, passing through the lower cervical region and the midline lower chest, along the white line, side-stepping in a watermelon slice shape the navel, the external genital organs and the mammary glands, until reaching the pubis. Next, four incisions should be made on the inner surface of each member, from the distal extremities to the inferomedial longitudinal incision. Subsequently, the proper skinning begins, using either sharp instruments or one's hands, while avoiding the perforation of the skin. The dog placed in a dorsal decubitus, the thoracic cavity is opened through the sectioning of both costal walls, whereas for animals placed in a lateral decubitus (i.e. large animals), the ribs, together with the sternum, are only sectioned on one side.

The heart was examined according to specific technical examination mammalian heart.

Initially, the pericardial sac must be opened through a buttonhole performed on the top of the heart, followed by two $V$ - shaped sections, from the apex towards the anterior and posterior sides of the heart. For the opening of the heart, two mandatory sections must be performed: one for the left side and one for the right side. In the case of small and average animals the heart can be held in hand, whereas for large animals (bulls, horses) the heart should be placed on a tray.

\section{Opening the left side of the heart}

While holding the heart in the left hand, with the anterior edge on the palm-top, a knife-incision must be performed on the posterior, most convex side of the heart (in the space between the right and the left pulmonary veins), from the wall of the left atrium to the apex. The atrial and ventricular cavities, as well as the left atrial-ventricular valves (bicuspid or mitral) must be emphasized.

\section{Opening the right side of the heart}

The heart must be positioned with the posterior edge on the palm-top of the left hand. Next, an incision must be performed on the anterior, most convex side of the heart, from the right atrium (in the space between the two cava veins), to the inter-ventricular ditch. The right atrialventricular valves, the atrial cavity and the right ventricular cavity must be emphasized [4].

\section{Results and discussions}

On opening the chest cavity was observed presence of a milky liquid in appreciable amounts (fig 1). Upon examination by splitting the right heart was observed in ventricle heartworms (fig.2). Although it is said that chilotorax is idiopathic, there is a correlation between chilotorax some heart problems listed above. If we did our study the link between chilotorax and heartwarm, Heartworm infection may cause heart failure that increases pressures in the vessels that the thoracic duct

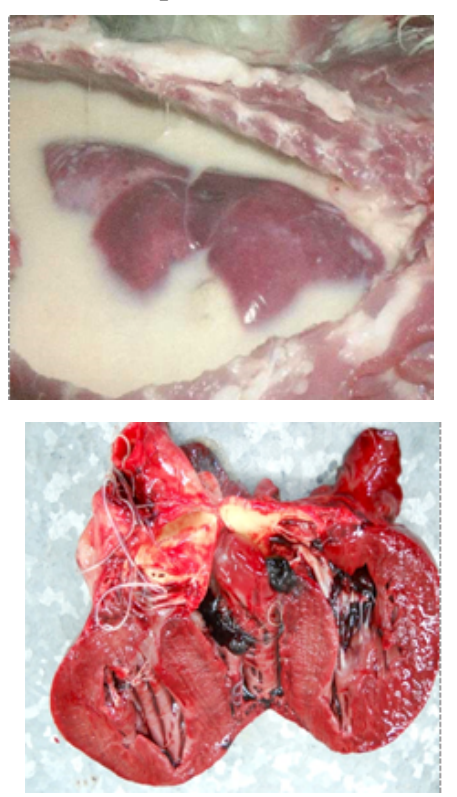

Fig. 1. Milky white fluid in the thoracic cavity (chylothorax)

Fig. 2. Heartworm,

Dirophylaria immitis

empties into. By compression on long-term lung was reached deadly. cardiorespiratory insufficiency $[5,13,17]$.

\section{Conclusions}

Chylothorax was diagnosed in a Afghan dog of 3 year, that presented in the necropsy a accumulation of lymphatic fluid in the chest cavity.

Although it is said that chilotorax is idiopathic, in this case is a correlation between chilotorax and heartwarm, wich represent cause heart failure that increases pressures in the vessels that the thoracic duct empties into.

Chilotoraxul long-term (chronic form) produces compression deadly cardiorespiratory failure.

Acknowledgements: This research work was carried out with the support of the project Dezvoltarea infrastructurii de cercetare, educape oi servicii în domeniile medicinei veterinare of tehnologiilor inovative pentru RO 05, cod SMIS-CSNR 2669.

\section{References}

1. ADRIAN STANCU, General veterinary pathological anatomy 2014, Editura Agroprint, ISBN - 978-606-8037-50-3, 
2. ADRIAN STANCU, Practicum of veterinary pathological anatomy, 2014, Editura Agroprint, ISBN- 978-606-8037-48-6.

3. ADRIAN STANCU, Special veterinary pathological anatomy, 2014, Editura Agroprint, ISBN - 978-606-8037-49-3.

4. ADRIAN STANCU, Diagnostic necropsic veterinar, 2013. Editura Mirton 2013, ISBN -978-973-52-1395-4.

5. BIRCHARDSJ , SMEAK DD, MCLOUGHLIN MA: Treatment of idiopathic chylothorax in dogs and cats. J Am Vet Med Assoc 212:652-657, 1998. 6. BREZNOCK EM: Management of chylothorax: Aggressive medical and surgical approach. Vet Med Report 1:380,382-384.

7. COTOI, O.S., SUCIU, M., PACURAR, M., ORMENISAN, A.,Rev. Chim. (Bucharest), 66, no. 2, 2015, 219

8. FARNSWORTH R, BIRCHARDS: Subcutaneous accumulation of chyle after thoracic duct ligation in a dog. J Am Vet Med Assoc 2016-2019, 1996; 208.

9. FOSSUM TW, BIRCHARD S , JACOBS RM: Chylothorax in 34 dogs. J Am Vet Med Assoc, 1986, 188:1315-1317.

10. FOSSUM TW, FORRESTER SD, SWENSON CL, MILLER MW, COHEN ND, BOOTHE HW, BIRCHARD S) : Chylothorax in cats: 37 cases (19691989). J Am Vet Med Assoc, 1991, 198:672-678.
11.FOSSUM TW, JACOBS RM, BIRCHARD S) : Evaluation of cholesterol and triglyceride concentrations in differentiating chylous and nonchylous pleural effusions in dogs and cats. J Am Vet Med Assoc, 1986, 188;49-51.

12. FOSSUM TW: The characteristics and treatments of feline chylothorax. Comp on Cont Ed, Sept 1998, 914-928.

13. GELZER ARM, DOWNS MO, NEWELL SM, MAHAFFEY MB, FLETCHER J, LATIMER KS: Accessory lung lobe torsion and chylothorax in an Afghan Hound. J Am Vet Med Assoc, 1997, 33:171176.

14. MARIUS ILIE, KALMAN IMRE, IONELA HOTEA, MIRELA IMRE, Patogeni ai sângelui în continua expansiune, Editura Agroprint \& Mirton Timisoara 2011.

15. MEADOWS RL, MACWILLIAMS PS: CHYLOUS effusions revisited. Vet Clin Pathol, 1994, 23:54-62.

16. PA'CA AURELIAN SORIN - Probleme de patologie renalã la câine, 2014, Ed. Ion Ionescu de la Brad lasi, ISBN 978-973-147-158-7, 153 pg.; (153pct).

17. THOMPSON MS, COHN LA, J ORDAN RC: Use of rutin for medical management of idiopathic chylothorax in four cats. J Am Vet Med Assoc, 1999, 15:345-348.

Manuscript received: 5.06 .2017 